\section{Primary gastric small-cell neuroendocrine carcinoma}

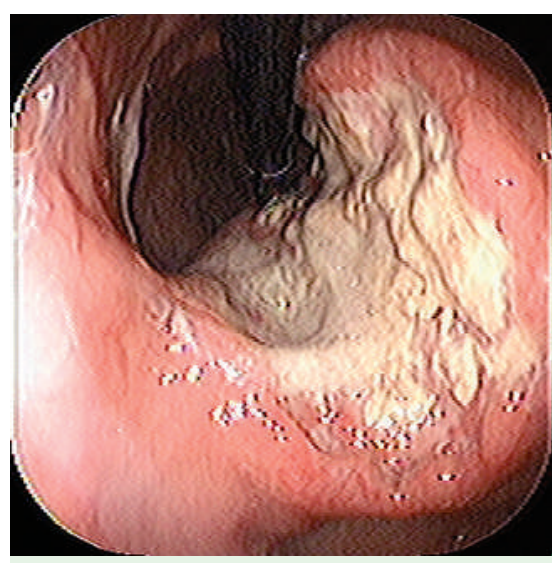

Figure 1 An endoscopic view (in retroflexion) showing a large ulcer with a necrotic base and irregular borders on the lesser curvature of the stomach.

Primary nonbronchogenic small-cell carcinoma has been decribed in a number of extrapulmonary sites, including the salivary glands, prostate, breast, esophagus, bladder, and cervix [1]. Primary smallcell gastric neuroendocrine carcinomas are extremely rare, representing less than $0.1 \%$ of all primary gastric cancers [2-5].

A 59-year-old woman presented with a 2month history of intermittent chest pain, nausea, dizziness, diaphoresis, and nonbloody emesis. She had lost $20 \mathrm{lb}$ in weight, but she said that she did not have early satiety or abdominal pain. Her hemoglobin level was $6.1 \mathrm{~g} / \mathrm{dL}$, with an iron saturation of $7 \%$. Computed tomography did not show any lung or pancreatic masses. Upper endoscopy revealed a 5$\mathrm{cm}$ ulcer with a necrotic base and friable, irregular borders on the lesser curvature of the stomach ( $\bullet$ Figure 1). Examination of biopsies of the gastric ulcer showed this to be a high-grade small-cell neuroendocrine carcinoma ( $\bullet$ Figures 2,3 ). Histologically, the malignant tumor showed solid, organoid, trabecular, and pseudoglandular patterns. The tumor cells showed a high nuclear grade, with minimal cytoplasm, indistinct nucleoli, and nuclear molding, and there was clear evidence of increased mitotic activity and necrosis ( Figure 3). Immunohistochemical staining, with adequate controls, showed the tumor cells to be strongly positive for pancytokeratin, synaptophysin, and CD56 ( $\bullet$ Figures $4-6$ ), but negative for chromogranin A. Staining for TTF-1, a tissue-specific transcription factor that is specific to thyroid, lung, and brain tissues [3] was negative. This combination of histopathological and immunohistochemical findings was compatible with a high-grade primary smallcell neuroendocrine carcinoma of the stomach. The patient declined further evaluation and management.

Gastric neuroendocrine carcinoma is one of the most aggressive cancers, and has an extremely poor prognosis. To date, only 38 cases have been reported (34 of them in Japan) since the first description of the tumor in 1976 [2,4]. Most cases have been described in male patients. Our patient is an African American woman from the United States, which makes this case even more interesting and unique. Because of the rarity of these cancers, the classification and biological characteristics of primary gastric neuroendocrine carcinomas remain controversial, and neuroendocrine differentiation is recognized only after appropriate immunohistochemical staining. The literature describes synaptophysin, chromogranin A, CD56, and other immunohistochemical markers as reliable for detecting neuroendocrine differentiation; and the CD56 marker is also being helpful for differentiating between small-cell and large-cell carcinomas [5].

\section{Acknowledgment} $\nabla$

The authors wish to thank Dr. Enrique Gomez from the Department of Pathology at the University of Texas-Houston for his assistance in the preparation and inter-
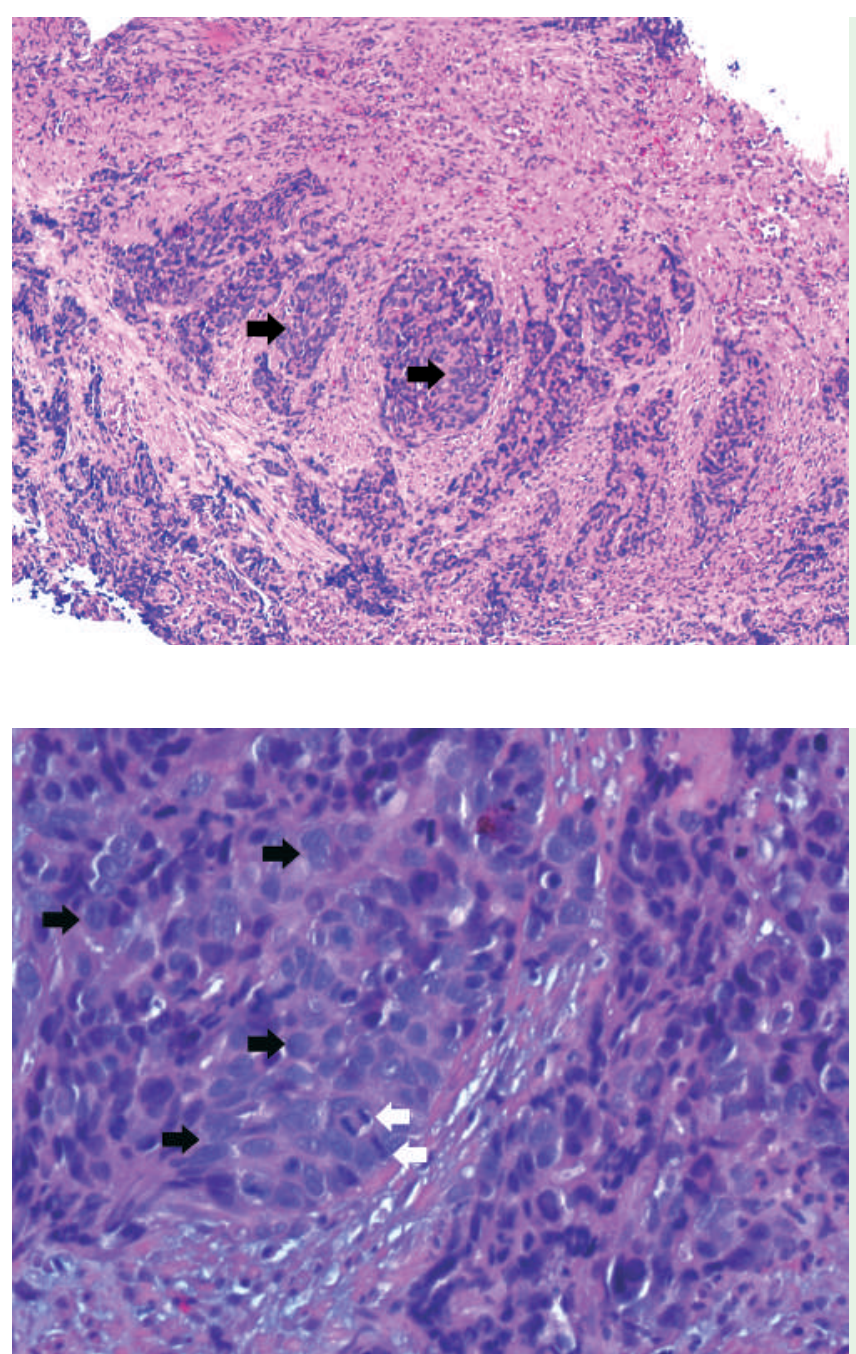

Figure 2 A low-power view of the gastric neuroendocrine carcinoma. The normal gastric mucosal architecture is nearly completely replaced by the tumor. The black arrows show constellations of malignant cells (hematoxylin and eosin [H \& E] stain, original magnification $\times 10$ ).

Figure 3 Microscopic view of the gastric neuroendocrine carcinoma, showing tumor cells with a high nuclear grade, with minimal cytoplasm, "salt and pepper"-like chromatin (black arrows), indistinct nucleoli, and nuclear molding. There is clear evidence of increased mitotic activity (white arrows). (H \& E stain, original magnification $\times 40$ ). 


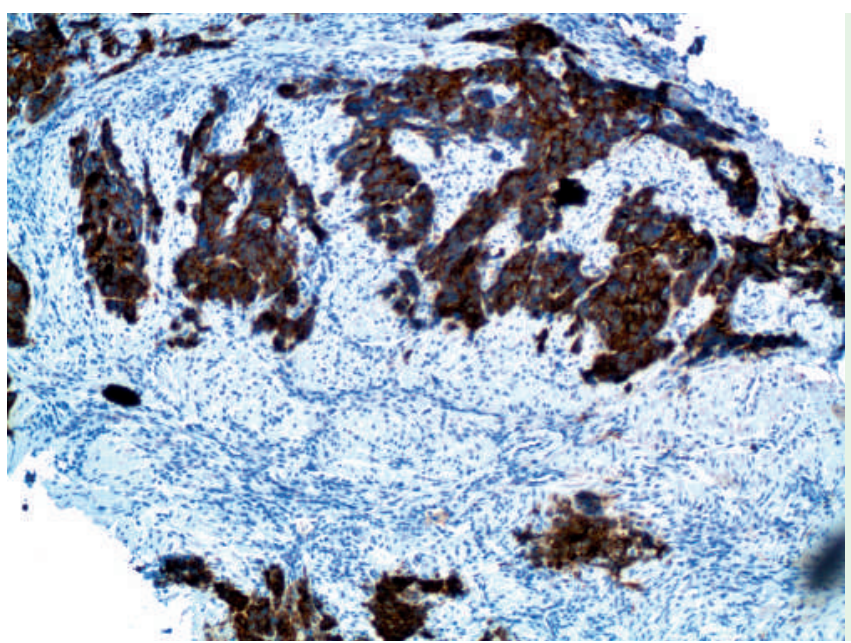

Figure 4 Microscopic view of the gastric neuroendocrine carcinoma biopsy specimen, showing positive staining for pancytokeratin (original magnfication $\times 10)$.
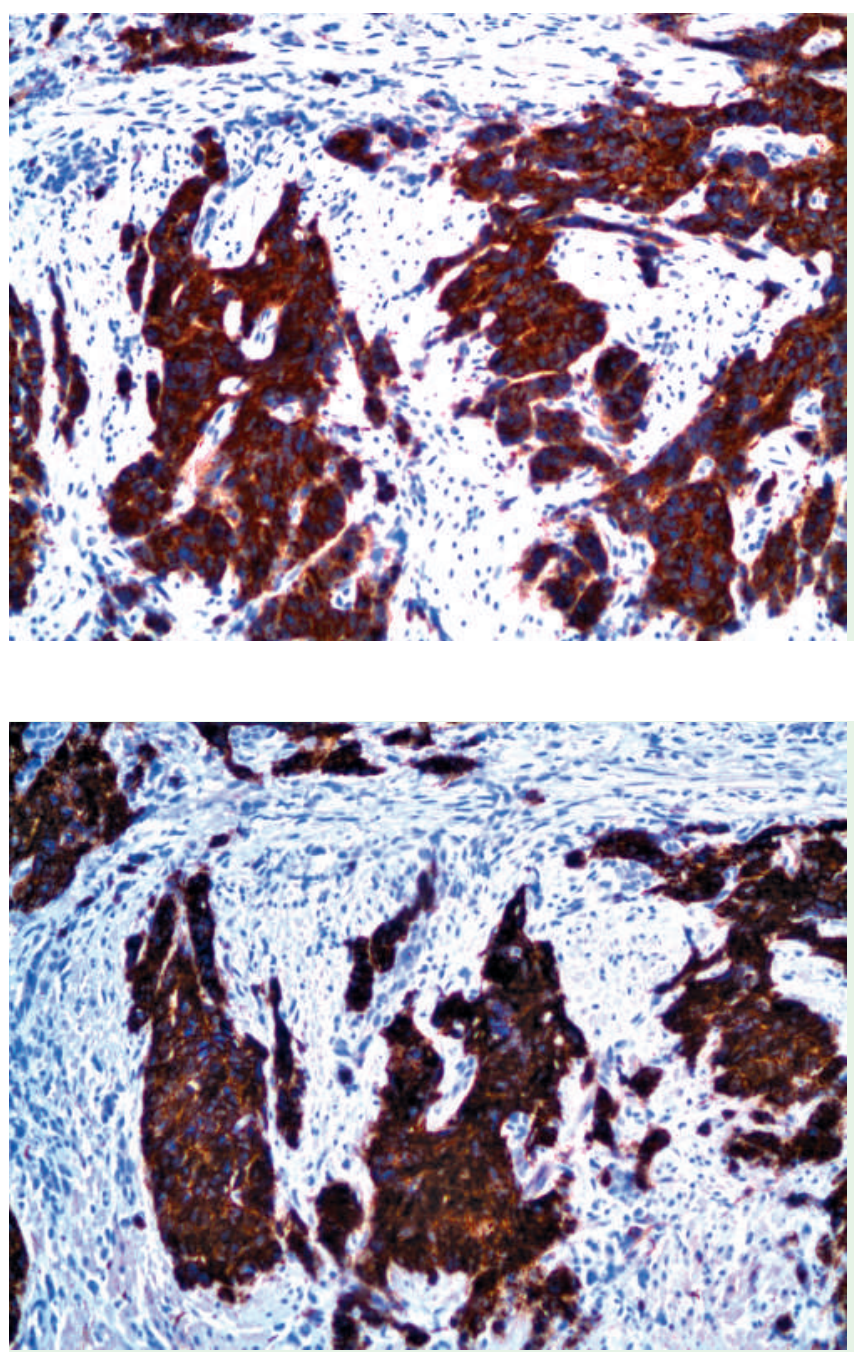

pretation of the histopathology specimens.

Endoscopy_UCTN_Code_CCL_1AB_2AD_3AB

\section{A. Shpaner, T. E. Yusuf}

Division of Gastroenterology,

The University of Texas Health Science

Center at Houston, Houston, Texas, USA

\section{References}

1 Ibrahim NB, Briggs JC, Corbishley CM. Extrapulmonary oat cell carcinoma. Cancer 1984; 54: 1645 - 1661

2 Kusayanagi S, Konishi K, Miyasaka $N$ et al. Primary small cell carcinoma of the stomach. J Gastroenterol Hepatol 2003; 18: $743-747$

3 Hiroshima K, Iyoda A, Shida T et al. Distinction of pulmonary large cell neuroendocrine carcinoma from small cell lung carcinoma: a morphological, immunohistochemical, and molecular analysis. Mod Pathol 2006; 19: 1358 - 1368

4 Matsusaka T, Watanabe H, Enjoji M. Oat cell carcinoma of the stomach. Fukuoka Igaku Zasshi 1976; 67: 65-73

5 Matsui K, Jin XM, Kitagawa $M$ et al. Clinicopathologic features of neuroendocrine carcinomas of the stomach: appraisal of small cell and large cell variants. Arch Pathol Lab Med 1998; 122: 1010-1017

\section{Bibliography}

DOI 10.1055/s-2007-966572

Endoscopy 2007; 39: E310-E311

(c) Georg Thieme Verlag KG Stuttgart · New York . ISSN 0013-726X

\section{Corresponding author \\ T. E. Yusuf, MD}

Division of Gastroenterology

The University of Texas Health Science Center at Houston

6431 Fannin Street, Suite 4.234

Houston

Texas 77030

USA

Fax: +1-713-500-6699

tony.yusuf@uth.tmc.edu 